\title{
Should we screen for intracranial aneurysms in children with autosomal dominant polycystic kidney disease?
}

\author{
Emma Y. X. Walker ${ }^{1} \cdot$ Matko Marlais $^{1,2}$ (D)
}

Received: 25 August 2021 / Revised: 9 December 2021 / Accepted: 10 December 2021 / Published online: 2 February 2022

(c) The Author(s), under exclusive licence to International Pediatric Nephrology Association 2022

\begin{abstract}
This is an overview of the challenges associated with screening for asymptomatic intracranial aneurysms (ICA) in children with autosomal dominant polycystic kidney disease (ADPKD). ADPKD is the most common inherited kidney disease affecting 1 in 1,000 people. ICAs are an extra-kidney manifestation of ADPKD, and while the exact pathophysiology of how they develop is unknown, we know that they more commonly occur in the adult rather than paediatric population. ICAs can be found in up to 9-11.5\% of adults with ADPKD, but ICA rupture remains a rare event in adults with an incidence of 0.04 per 100 patient years. ICA size is an important factor in determining the risk of aneurysm rupture and therefore affects the decision on intervention in asymptomatic adults. For some, unruptured aneurysms cause no clinical significance, but those that rupture can be associated with devastating morbidity and mortality. Therefore, if detected, the treatment for unruptured ICAs is usually endovascular coiling, alongside recognising the importance of preventative interventions such as hypertension management. There are, however, no current guidelines for either adult or paediatric patients with ADPKD supporting regular screening for asymptomatic ICAs, although there is a suggestion for individualised practice, for example, with those with a positive family history. The UK clinical guidelines for ADPKD in children make research recommendations due to a lack of published literature, which in itself indicates that ICA rupture is an extremely rare phenomenon in children.
\end{abstract}

Keywords Autosomal dominant polycystic kidney disease $\cdot$ ADPKD $\cdot$ Intracranial aneurysms $\cdot$ Screening $\cdot$ Paediatrics

$\begin{array}{ll}\text { Abbreviations } \\ \text { ADPKD } & \begin{array}{l}\text { Autosomal dominant polycystic kidney } \\ \text { disease }\end{array} \\ \text { GA } & \begin{array}{l}\text { General anaesthetic } \\ \text { Intracranial aneurysms }\end{array} \\ \text { ICA } & \begin{array}{l}\text { Kidney Disease Improving Global Outcomes } \\ \text { KDIGO }\end{array} \\ \text { KRT } & \text { Kidney replacement therapy } \\ \text { MRA } & \text { Magnetic resonance angiography } \\ \text { NPV } & \text { Negative predictive value } \\ \text { PPV } & \text { Positive predictive value } \\ \text { RCT } & \begin{array}{l}\text { Randomised control trial } \\ \text { Subarachnoid haemorrhage }\end{array} \\ \text { SAH } & \text { Time of flight magnetic resonance } \\ \text { TOF MRA } & \begin{array}{l}\text { angiography } \\ \end{array}\end{array}$

Matko Marlais

m.marlais@ucl.ac.uk

1 Department of Paediatric Nephrology, Great Ormond Street Hospital for Children NHS Foundation Trust, Great Ormond Street, London WC1N 3JH, UK

2 UCL Great Ormond Street Institute for Child Health, London, UK

\section{Introduction}

Autosomal dominant polycystic kidney disease (ADPKD) is the most common hereditary kidney disorder, estimated to affect 1 in 1,000 people [1, 2]. ADPKD is characterised by the development and expansion of multiple kidney cysts in both kidneys and can eventually progress to chronic kidney disease stage 5 requiring kidney replacement therapy (KRT). Furthermore, it is a ciliopathy and therefore a systemic disorder, and so it is associated with extra-kidney manifestations including vascular abnormalities such as intracranial aneurysms (ICA), as well as polycystic liver disease, cardiac valvular involvement, and pancreatic cysts [2].

ICAs are a rare phenomenon in paediatric patients. Indeed, patients with ADPKD who develop ICAs generally do so well into adulthood [3]. Despite this, given the significant morbidity and mortality associated with ICA rupture, parents of children with ADPKD often have notable anxiety about the risk of ICA rupture. The recently published outcomes of the SONG-PKD consensus workshop highlight the fact that cerebral aneurysms are a significant source of worry for some patients with ADPKD, despite them being a rare 
event [4]. As a result, paediatric nephrologists are frequently asked whether to screen children for ICAs, often by worried family members who may have had a relative with ADPKD and a history of ICA rupture. This prompts a dilemma for the clinician: to screen or not to screen?

This review aims to consider the topic of ongoing debate around screening for asymptomatic ICAs in patients with ADPKD.

\section{Aetiology, pathophysiology and clinical significance:}

The majority of ADPKD cases are secondary to mutations in the genes PKD1 (85\%) and PKD2 (15\%). These mutations are inherited in a Mendelian autosomal dominant fashion, and therefore, individuals at risk have a 50\% chance of inheriting the disease. An additional 10\% of cases are caused by de novo mutations [2]. The phenotype of ADPKD varies greatly in clinical severity, from newborns with large cystic kidneys to adults with preserved kidney function.

$P K D 1$ and $P K D 2$ encode for the proteins polycystin- 1 and polycystin-2 respectively. Polycystin proteins are expressed on many tissues, including vascular smooth muscle cells and the endothelial cells that make up the vascular wall $[5,6]$. In vivo experiments, such as those in mice, targeted mutations in either $P K D 1$ or $P K D 2$ results in haemorrhages, aneurysm formation and defects in vessel branching, thus supporting the important role the polycystin proteins have on developing and maintaining the vascular system [6].

Although several protein-protein biochemical interactions have been ascribed to polycystins, the exact cellular pathways disrupted by changes in the polycystin proteins remain uncertain [7]. What is known is that mutations in $P K D 1$ and $P K D 2$ lead to a reduction in intracellular calcium and therefore alter the calcium homeostasis [2]. This is demonstrated in a mouse model, whereby haploinsufficiency for PKD2 resulted in more intracranial vascular abnormalities [6]. Furthermore, as mentioned earlier, ADPKD is a ciliopathy and again mouse models have suggested that in the epithelium of the kidney, there are cilia with polycystin complexes. The exact pathway is unknown, but likely these cilia function through a combination of chemo-transduction and mechano-transduction pathways. They respond to the shear stress of fluid, again altering calcium signalling and subsequently contributing to tissue remodelling [8].

The combination of altered polycystin expression with the traditional inflammatory insults associated with impaired kidney function and other risk factors such as hypertension and smoking, which cause haemodynamic stress, give rise to vascular remodelling and increased aneurysm formation [1].

The clinical significance of ICA development varies; many patients will be completely unaffected and may not know about the existence of an ICA. However, for those that rupture, the outcome can be devastating with a significant percentage experiencing morbidity, such as significant neurodisability, or mortality.

\section{ICA in Adults with ADPKD}

\section{Background}

It has been known for 30 years that ICAs are more common in adults with ADPKD than the general population and therefore it is worth reviewing the adult practice first [9]. The overall prevalence of unruptured ICAs in adults, in the general population, without co-morbidities is estimated at $3 \%[3,10]$, whereas the prevalence of unruptured ICAs is approximately threefold greater at $9-11.5 \%$ in adults with ADPKD [11, 12].

There is familial clustering of ICAs demonstrated in the literature; indeed, the risk of developing an ICA if one has a positive family history is at least 2 times greater than without: the prevalence of unruptured ICAs in those without a family history is approximately $6 \%$ compared to $14-16 \%$ with a family history $[13,14]$. The risk of developing an ICA increases further, albeit to a lesser extent with other environmental and non-genetic factors, including age over 30 , a previous aneurysm, hypertension and smoking [3, 12].

Aneurysm rupture itself is estimated at approximately 0.04 per 100 person years, which is approximately 5 times higher than the general population $[12,14]$. On average, ICA rupture in those with ADPKD occurs around the age of 40 years, which is 10 years earlier than the general population [14-16]. Aneurysm ruptures can cause subarachnoid haemorrhages (SAHs), and subsequently significant neurodisability and death. In those with ADPKD, rupture is associated with a $35-55 \%$ morbidity and mortality, which is not dissimilar to the general population [14, 15, 17]. However, the fact that aneurysm ruptures occur earlier in patients with ADPKD means that the years of life lost are greater.

One of the largest international studies, the ISUIA (International Study of Unruptured Intracranial Aneurysms), demonstrated an increased risk of ICA rupture, in all causes of ICAs, if the size is $>7 \mathrm{~mm}$ and located in the posterior circulation [18]. This has been subsequently confirmed with systematic reviews and meta-analyses [3]. The KDIGO report from 2015 states asymptomatic screening for ICAs in those with ADPKD reveals $80-90 \%$ of those with ICA are in the anterior circulation and nearly all are $<7 \mathrm{~mm}$ [16]. However, rupture of ICAs can still occur for those that are small and located in the anterior circulation and therefore a single risk factor cannot be used in isolation when assessing aneurysms identified is not solely dependent on size or location [10]. 
Increased size, location to the posterior circulation and previous SAH are risk factors for ICA rupture. However, family history must also be discussed in the context of ICA rupture. The risk of SAH is greater if first degree relatives with ADPKD have also previously had an ICA rupture; however, the rates are similar between those with ADPKD and the general population when comparing second degree relatives that are affected [12]. Twin studies often provide unique insight into 'nature vs. nurture'. A study in which monozygotic twins living non-concomitantly observed that one developed a SAH at the age of 20, and the other twin remains stroke-free at the age of 48 [15]. This gives rise to the question of to what extent many years of shared environment and other non-genetic factors contributes to ICA formation and rupture, and whether this outweighs the genetic component [1]. For paediatric cases, it is unlikely that they will have had long enough to share an environment or be exposed to non-genetic factors for ICA development and rupture risk. Therefore, family history of aneurysm rupture alone may not be enough of a reason to screen children with ADPKD for ICA.

\section{Investigations and treatment}

As asymptomatic ICAs are not routinely screened, there is no standardised screening protocol. Traditionally, if ICAs were being screened, the most common diagnostic techniques were magnetic resonance angiography (MRA) and four-vessel interventional angiography, requiring iodinated contrast media. However, 1 in 4 (25\%) experienced transient complications as a result. These included worsening of kidney function, arterial spasm/dissection and visual symptoms. This is compared to $1.3 \%$ in the general population [11]. Following recent studies, it is now recommended to use a non-contrast time of flight MRA (TOF MRA) to screen for ICAs, without gadolinium enhancement, which has a similar sensitivity but without the radiation exposure and nephrotoxic contrast [19].

Symptomatic ICAs, those associated with sudden onset headaches or neurological symptoms in a patient with or without ADPKD, will need urgent neurological imaging. Further discussion of this is, however, outside the scope of this review [6].

Preventative interventions for the management of ICAs should not be ignored. These include addressing modifiable risk factors such as smoking, alcohol intake and hypertension management. This is a view supported by patients themselves, in which they recognise the benefits of moving kidney disease interventions upstream rather than waiting for downstream complications to occur [20].

If invasive management of an unruptured ICA in adults is to be considered, a multi-disciplinary team approach should be adopted, involving nephrologists, neurosurgeons and interventional radiologists. Interventional options include endovascular embolisation and surgical clipping. However, a randomised control trial of patients in the general population, i.e. not limited to patients with ADPKD, demonstrated reduced morbidity and mortality with endovascular embolisation compared to surgical clipping. Therefore, this is the preferred treatment approach in adults [21].

Although ruptured ICAs themselves carry around a 50\% combined morbidity and mortality rate, treatment of unruptured ICAs is not without significant risks. Risks include death, intracranial haemorrhage, thromboembolic events and vessel perforation/dissection [11,22]. There is a still a combined morbidity (defined as dependency) or mortality of $23.7 \%$ after endovascular coiling and $30.6 \%$ after surgical clipping [21]. Moreover, these risks of iatrogenic haemorrhage or infarction are reportedly increased in those with ADPKD compared to the general population [23]. In addition, the endovascular approach has ongoing concerns regarding the durability of coil embolisation and incomplete occlusion $[14,21]$.

\section{Current guidelines}

Screening for asymptomatic ICAs in ADPKD has been discussed for nearly 30 years, yet there is still no clear consensus [24]. Adult nephrologists across Europe have divergent practices with $28 \%$ advocating systematic screening and over $90 \%$ screening high-risk cases (e.g. family history of ICA rupture, sudden death, stroke and migraine) [25].

A decision tree analysis in the 1990s recommended routine MRAs in patients with ADPKD, particularly those with a family history of ICAs, as the resultant treatment can expect to add some 10 years to a patient's life if they are 20 years old [26]. Further, a recent analysis from 2018 suggested that screening is cost-effective and argues that screening should therefore be performed in all patients, and not just those with a known familial risk, as this confers the highest gain in quality-adjusted life years (QALY) [13].

Yet, the 2018 analysis highlights a dilemma that occurs with screening: a number of small de novo aneurysms could be detected, which, in turn, will likely be managed nonoperatively but will require monitoring [13]. This has the potential to cause elevated anxiety for the patient. This study therefore proposed that a prospective study with a longer duration of follow-up be conducted and the impact on mental health reviewed.

On the other hand, the KDIGO consensus guidance is not supportive of widespread screening for ICAs in those with ADPKD. However, they do acknowledge that different attitudes exist and they do support addressing modifiable risk factors [16]. 
If the decision is made to screen, the next questions would be when to intervene and at what interval should we repeat imaging?

If upon screening an ICA is found, there must then be a framework in place to decide at which point to treat it. A hypothetical scenario (non-ADPKD) showed that one can only expect additional quality-adjusted life years and costeffectiveness in those treated if the ICA was $\geq 10 \mathrm{~mm}$ in diameter [14]. But the majority of ICAs found on screening and in cohort studies are less than $5 \mathrm{~mm}$ in diameter [22].

A prospective study showed that only 2 of 76 patients with initially negative MRA scans developed an ICA when rescreened approximately 10 years later. This result suggests rescreening those with positive family history with initial negative scans at 10-year intervals [27]. However, another study describes 45 patients with ICAs, 11 of whom presented with SAH, of which 7 had known ADPKD and 2 had negative screening just 3-5 years earlier [28]. Therefore, screening will never completely negate the risk of ICA rupture, and the frequency of screening intervals in adults with ADPKD would require an extensive debate which is beyond the scope of this review.

\section{ICA in children with ADPKD}

\section{Background}

ICAs in children account for 5-6\% of the total aneurysms of the whole population [10, 29]. Within the paediatric population, ICAs in general have a number of aetiologies and are actually much more heterogenous in nature: for example, cystic fibrosis, tuberous sclerosis, Marfan's syndrome and infections. ADPKD is therefore just one uncommon cause for ICA in children [29].

Given the more varied aetiology of ICAs in children compared to adults, there is also a difference in characteristics of the aneurysm in the general paediatric population. The mean aneurysm size is smaller and more frequently located in the middle cerebral artery; however, there are significantly more 'giant' aneurysms ( $>25 \mathrm{~mm}$ ) which tend to be found in the posterior circulation [29-32]. This highlights the unique nature of paediatric aneurysms and how we cannot simply generalise adult findings to the paediatric population.

The true prevalence of ICAs and their size and location in children with ADPKD are unknown, and there is little description of ICAs in children with ADPKD specifically; this absence of published data is reassuring in that this is a very rare condition.

Moreover, there are only a few case reports of ICA ruptures and $\mathrm{SAH}$ in children with ADPKD despite there being hundreds of thousands of children with the condition worldwide [33-36]. The age range spans the whole of the paediatric age group, with the youngest of these being described at just a few days old, and in this case it is the presenting manifestation of ADPKD [33]. While we know the risk of ICA development increases with age into adulthood, it is unclear whether the risk significantly increases through childhood into adolescence. However, the 1994 study of 77 patients with ICA did show 7 had aneurysm rupture $<21$ years of age with the youngest aged 15 years [15].

We do not know the natural history of ICA development in children. Furthermore, the modifiable risk factors which we know exist in adulthood, such as smoking and alcohol consumption, do not typically exist for our paediatric population. However, the control of hypertension is an important modifiable risk factor in children and the risk of hypertension in ADPKD rises with increasing age even in adolescence. Addressing this would likely impact more on prevention of progression of chronic kidney disease than ICA rupture [37, 38].

Children with a first degree relative with ICA rupture are presumably at higher risk, but there are no direct data on whether family history is relevant in childhood; as mentioned earlier, the environmental and other non-genetic risk factors which are associated with adult ICA rupture risk may mean a family history is less relevant in children.

\section{Investigation and Management}

Given the research in adults, the imaging tool of choice for investigating for an asymptomatic ICA in children would be TOF MRA. This has a high sensitivity for detecting small aneurysms $(67 \%<3 \mathrm{~mm}, 79 \%<5 \mathrm{~mm}, 95 \%>5 \mathrm{~mm})$ and moreover, does not require contrast, nor has the associated risk of radiation such as that associated with a CT [16, 39]. However, for the younger children, they would also require sedation or a general anaesthetic (GA) to be able to obtain images of sufficient quality. This in itself is not without associated risks of GA (albeit they are low) [40, 41].

Generally, the larger aneurysms will be treated, as these are thought to be more likely to rupture and we know that giant aneurysms are more common in children compared to adults [42, 43]. However, there is a case report of a 4-year-old with ADPKD who had an aneurysm rupture, and it was only $3 \mathrm{~mm}$ [34]. Therefore, size again is only one factor used in the decision over whether to surgically intervene or not. This 4-year-old did, however, have hypertension, emphasising the importance of good blood pressure control which is a priority for the overall management of ADPKD in childhood [44].

As with adults, if intervention is to be carried out, then it should be performed as part of the multi-disciplinary team with nephrologists, neurosurgeons and interventional radiologists. Moreover, given that these interventions are rarer in children than in adults, any intervention should be carried out in a centre of excellence with experience of interventional neurovascular treatment in children [45]. 
The International Subarachnoid Aneurysm Trial in 2002 was the previously cited RCT that compared endovascular coiling with neurosurgical clipping for treatment of ruptured ICAs. It found that the outcome in terms of survival free of disability at 1 year is significantly better with endovascular coiling [21] and this is therefore also the treatment of choice for children as evidenced in a paediatric-specific analysis [46].

Again, there are risks associated with endovascular coiling and with surgical clipping. In addition to those seen in adults, the aforementioned more complex nature of paediatric ICAs (e.g. giant size) pose a larger challenge and a higher demand for durability of treatment. Endovascular coiling may therefore not be sufficient to achieve longstanding occlusion and may even need, for example, parent artery occlusion [43]. Furthermore, many of these are from ruptured cases; there are even fewer data on the treatment of unruptured ICAs in children.

\section{Screening recommendations}

As rupture of ICA is an exceedingly rare complication in childhood, and as there is no clear treatment or intervention guidelines for unruptured ICA in children, there is widespread opinion that routine screening in asymptomatic children is not justified $[44,47]$. We must also remember that screening will likely include sedation or a general anaesthetic in order to obtain the MRA imaging, which itself is not without risks.

The current UK guidelines suggest more data are needed first before recommendations can be made and these include:

- determining the incidence of SAH and the prevalence of ICA in children with ADPKD.

- determining whether screening for ICAs reduces the risk of intracranial events [47].

However, in rare cases with a positive family history and a strong desire to ease anxiety by screening, an individualised approach may be justified [44]. Though, we must be aware that anxiety is likely to be significantly increased for families in the hypothetical situation where a very small aneurysm is found and simply monitored as the opinion of the multidisciplinary team is that intervention is not justified.

\section{Pitfalls of diagnostic test accuracy statistics in ultra-rare prevalence disorders}

A good screening programme has a number of criteria to address. Firstly, the disease itself. There must be sufficient prevalence and morbidity in the target population. One could argue that ICAs are a rare disorder, and ultra-rare in the population of children with ADPKD.
Secondly, the investigation to determine the disease/disorder should be efficient, sensitive, specific and safe. We know that 3D TOF MRA has a specificity of over $75 \%$ and average sensitivity of nearly $90 \%$, though it is more sensitive with larger-sized aneurysms compared to smaller ones [39].

Finally, treatment should be efficient, safe, cost-effective and lead to improved outcomes with earlier intervention.

With this in mind, a few questions need to be addressed before routine screening becomes part of routine clinical care.

Firstly, if the first scan is negative, when should rescreening be offered? Ten years is proposed in the adult population, although some cases would be missed even with 5 yearly screening, so what about in the paediatric population?

When looking at screening tests, we need to assess 4 metrics to assess their adequacy for clinical practice. Specificity and sensitivity look at how effective the test is and, in this case, how effective the TOF-MRA is at detecting ICAs, whereas the positive predictive value (PPV) and negative predictive value (NPV) indicate, respectively, the likelihood that the test-the imaging — will successfully identify whether the patient does or does not have the ICA [48]. Importantly, while sensitivity and specificity are not affected by the prevalence of a disorder, PPV and NPV are.

Because screening tests are not part of routine clinical practice, we do not know the NPV or PPV. However, with a low-prevalence disorder, such as ICAs in children with ADPKD, the PPV will be low, too. Moreover, even if the specificity of the test, the MRA, is high, e.g. $99 \%$, there may still be many more false positives than true positives in an ultra-rare condition. It is therefore possible to imagine a situation where a false positive was found on MRA and intervention performed, only to later find that it was a false positive. Given the not insignificant risk of neurovascular intervention, it could result in the devastating situation where a child suffers harm from a neurovascular intervention which was not required, due to a false positive on MRA.

This leads to what to do when an abnormality on MRA is found. Firstly, there are no clear guidelines as to when to perform neurovascular intervention. As described, even an aneurysm of $3 \mathrm{~mm}$ has resulted in rupture and therefore, size is not necessarily the only indicator. However, not intervening could also have a negative impact due to the anxiety it may cause with the knowledge that a child has an untreated ICA.

\section{Decisions to screen for asymptomatic unruptured ICA in children with ADPKD on a population level}

Should we screen for asymptomatic unruptured ICA in children with ADPKD on a population and health system level? Some may argue for screening, given that the 
morbidity and mortality outcome of an ICA rupture is so high, but that is not a commonly-held view. A number of important factors must be considered.

One in 1,000 people have ADPKD. If we were to screen all these patients, that would be a significant number of extra MRA scans, many of which may need to be performed under general anaesthetic. This would require a huge amount of resource and considering the strain that many healthcare systems are under at present, and will be following the COVID-19 pandemic, this is unlikely to be feasible or cost-effective.

Moreover, there are no published data allowing us to assess the number needed to treat to prevent just one rupture of ICA in a child with ADPKD. Giving how rare the condition is, it is likely to be an extremely large number.

Given that the performance of a screening test is affected by the prevalence of a condition, some have proposed screening children with ADPKD and a family history of ICA rupture. This would theoretically increase the pre-test probability of finding a true result, but in reality, there are very little data to support that approach in childhood. This is especially true when we consider, as earlier, the risk of ICA rupture may in fact be due more to many years of shared environment ('nurture') rather than genetic susceptibility.

Screening patients for diseases is a balance of risks and benefits. In this scenario, the potential benefit is obvious: preventing a catastrophic intracerebral event which has wellestablished associated morbidity and mortality. However, the risks of screening are not to be dismissed: there is the risk of radiation, the risks of intervention if an unruptured ICA is found (particularly given that the unruptured ICA may never in fact rupture), and the anxiety to the child and family if an ICA is detected, but not treated.

\section{Conclusions}

ICAs are well known to be more prevalent in adults with ADPKD, compared to the general population. Identified risk factors for development and rupture of these ICA are family history, age and modifiable factors such as hypertension, smoking and alcohol consumption. Endovascular coiling is the treatment of choice for ICAs; however, it is associated with its own risks. Guidelines in adults do not support widespread screening but do offer suggestions of screening those at increased risk, such as those with positive family history.

There is a real lack of published data on ICAs in ADPKD in the paediatric population, but this lack of data suggests that this is an ultra-rare phenomenon. At present, most of the evidence for ICA in ADPKD in the paediatric population is based on case reports, or expert opinions, which are difficult to use to make good recommendations. Moreover, while we can learn from the adult literature, there are differences between the two populations and thus we cannot generalise all adult findings to children. Therefore, further data is needed to make a firm evidencebased recommendation on screening for unruptured ICA in children with ADPKD. Results that would help include, within the ADPKD paediatric population: the incidence of SAH, prevalence of unruptured ICAs and most crucially whether screening for ICAs would significantly decrease the risk of intracranial events.

Arguably, not screening for ICAs leaves a small but definite risk of rupture. However, this needs to be balanced with the risk of screening and the risk of associated intervention. Thus, at present there is no scientific justification to support generalised screening for unruptured ICA in children with ADPKD. We would also argue, for the reasons outlined above, that it is difficult to find scientific justification to even screen children who are at perceived increased risk of ICA rupture due to their family history. However, it is reasonable, and supported by international guidelines, that an individualised approach should be made. Figure 1 shows a flowchart to aid clinical decision-making. Targeting the modifiable risk factors, such as optimising blood pressure, is an important step in management. But it is essential to be mindful of the anxiety that some families have surrounding ICAs, for they may have had a previous negative experience. If families are adamant that they want to go ahead with screening, this can put clinicians in a difficult position. They should be carefully counselled about the significant risk of false positives with screening for an ultra-rare disorder, as well as the consequences this may entail with the potential for increased anxiety and the potential for neurovascular intervention on what may in fact be a false positive on MRA imaging. These concepts can be difficult for many families to understand, and therefore an extended discussion with adequate time for families to consider all options is important, to ensure they can make the right decision supported by their clinical team.

\section{Key summary points}

- Adults and children with ADPKD and acute neurological symptoms should undergo urgent evaluation for ICAs

- The pathophysiology of ICA development in those with ADPKD is unknown

- ICA rupture in the adult population is uncommon, and the incidence of ICA rupture in the paediatric population is ultra-rare 
Fig. 1 Flowchart to aid clinical decision making when seeing a child with ADPKD and considering the possibility of intracranial aneurysms. Abbreviations: ADPKD, autosomal dominant polycystic kidney disease; ICA, intracranial aneurysm

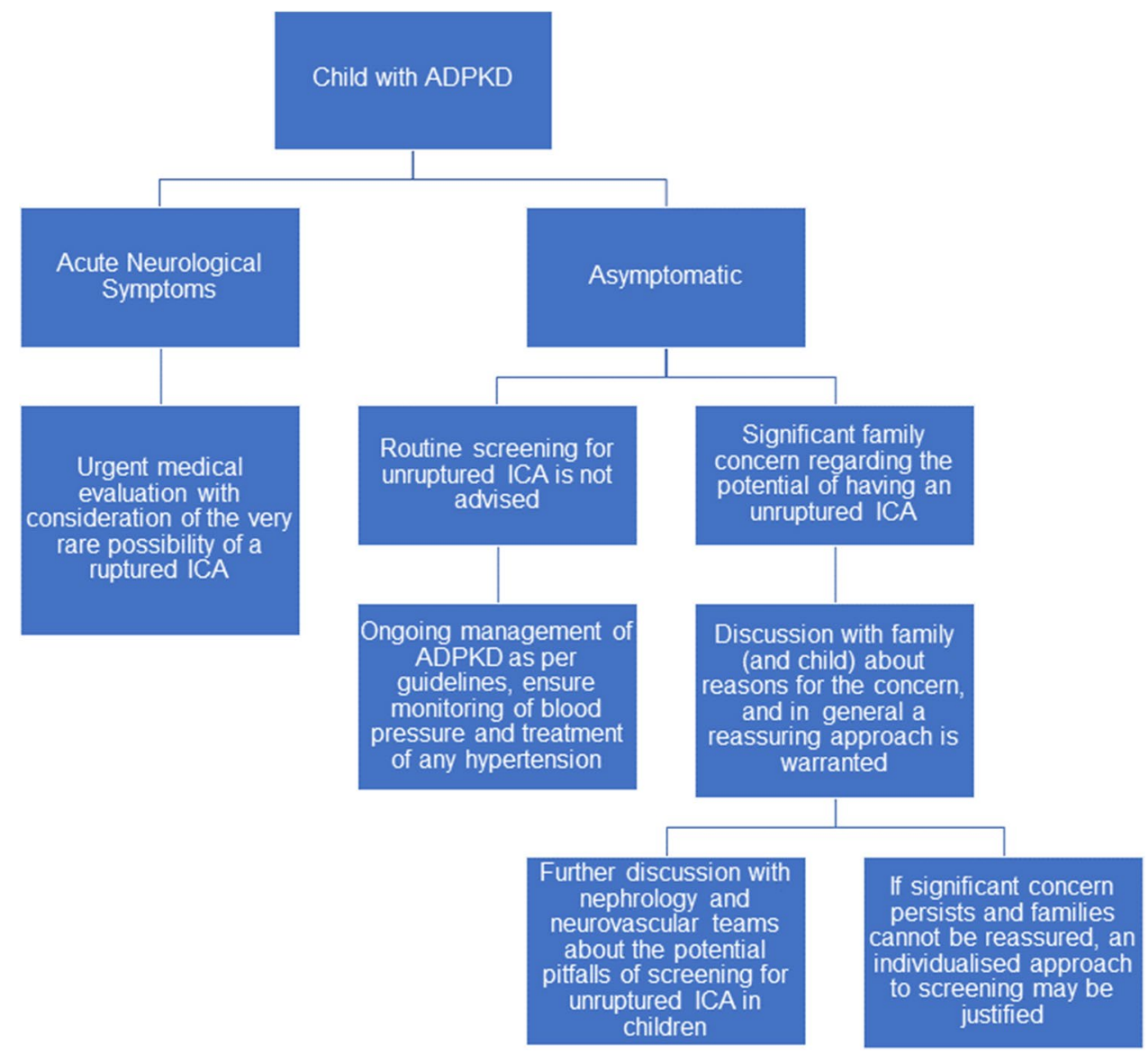

- ICA rupture is associated with significant morbidity and mortality; however, screening and intervening for unruptured ICAs is not without its own risks

- Positive family history of ICA rupture in isolation is not an absolute indication to screen children for ICAs, but rather an individualised approach should be sought and families should be carefully counselled

- We need to understand further the prevalence of ICA in children with ADPKD and whether screening would affect the outcomes, before being able to offer any evidence-based guidelines

\section{Multiple choice questions (answers provided following the reference list):}

1. What gene contributes to the majority of ADPKD cases?
a. PKD1
b. PKD2
c. PKD3
d. CYS1
e. CYS2

2. What is the average age of ICA rupture in those with ADPKD?
a. 12 years
b. 20 years
c. 34 years
d. 40 years
e. 52 years

3. What is the true prevalence of ICA in the ADPKD paediatric population?
a. $0.1 \%$
b. $1 \%$
c. $2 \%$
d. $5 \%$
e. unknown 
4. What is the imaging tool of choice for investigations for an ICA in children?
a. CT
b. X-ray
c. TOF MRA
d. Cranial ultrasound
e. PET scan

5. Which of the following statistical measures are affected by the prevalence of a disorder? ( 2 correct)
a. Sensitivity
b. Specificity
c. Positive predictive value
d. Negative predictive value

\section{Declarations}

Conflicts of interest The authors have no relevant financial or nonfinancial interests to disclose.

\section{References}

1. Kuo IY, Chapman A (2019) Intracranial Aneurysms in ADPKD: How Far Have We Come? Clin J Am Soc Nephro 14:1119-1121. https://doi.org/10.2215/cjn.07570719

2. Chebib FT, Torres VE (2016) Autosomal Dominant Polycystic Kidney Disease: Core Curriculum 2016. Am J Kidney Dis 67:792-810. https://doi.org/10.1053/j.ajkd.2015.07.037

3. Vlak MH, Algra A, Brandenburg R, Rinkel GJ (2011) Prevalence of unruptured intracranial aneurysms, with emphasis on sex, age, comorbidity, country, and time period: a systematic review and meta-analysis. Lancet Neurol 10:626-636. https://doi.org/10. 1016/s1474-4422(11)70109-0

4. Cho Y, Tong A, Craig JC, Mustafa RA et al (2021) Establishing a Core Outcome Set for Autosomal Dominant Polycystic Kidney Disease: Report of the Standardized Outcomes in NephrologyPolycystic Kidney Disease (SONG-PKD) Consensus Workshop. Am J Kidney Dis 77:255-263. https://doi.org/10.1053/j.ajkd. 2020.05.024

5. Ong ACM, Harris PC (2005) Molecular Pathogenesis of ADPKD: The polycystin complex gets complex. Kidney Int 67:1234-1247. https://doi.org/10.1111/j.1523-1755.2005.00201.x

6. Perrone RD, Malek AM, Watnick T (2015) Vascular complications in autosomal dominant polycystic kidney disease. Nat Rev Nephrol 11:589-598. https://doi.org/10.1038/nrneph.2015.128

7. Hardy E, Tsiokas L (2020) Polycystins as components of large multiprotein complexes of polycystin interactors. Cell Signal 72:109640. https://doi.org/10.1016/j.cellsig.2020.109640

8. Nauli SM, Alenghat FJ, Luo Y, Williams E et al (2003) Polycystins 1 and 2 mediate mechanosensation in the primary cilium of kidney cells. Nat Genet 33:129-137. https://doi.org/10.1038/ ng 1076

9. Chapman AB, Rubinstein D, Hughes R, Stears JC et al (1992) Intracranial aneurysms in autosomal dominant polycystic kidney disease. N Engl J Med 327:916-920. https://doi.org/10. 1056/nejm199209243271303

10. Etminan N, Rinkel GJ (2016) Unruptured intracranial aneurysms: development, rupture and preventive management. Nat Rev Neurol 12:699-713. https://doi.org/10.1038/nrneurol.2016. 150

11. Cagnazzo F, Gambacciani C, Morganti R, Perrini P (2017) Intracranial aneurysms in patients with autosomal dominant polycystic kidney disease: prevalence, risk of rupture, and management. A systematic review. Acta Neurochir 159:811-821. https://doi.org/ 10.1007/s00701-017-3142-Z

12. Sanchis IM, Shukoor S, Irazabal MV, Madsen CD et al (2019) Presymptomatic Screening for Intracranial Aneurysms in Patients with Autosomal Dominant Polycystic Kidney Disease. Clin J Am Soc Nephrol 14:1151-1160. https://doi.org/10.2215/cjn.14691218

13. Flahault A, Trystram D, Nataf F, Fouchard M et al (2018) Screening for intracranial aneurysms in autosomal dominant polycystic kidney disease is cost-effective. Kidney Int 93:716-726. https:// doi.org/10.1016/j.kint.2017.08.016

14. Pirson Y, Chauveau D, Torres V (2002) Management of cerebral aneurysms in autosomal dominant polycystic kidney disease. $\mathrm{J}$ Am Soc Nephrol 13:269-276

15. Chauveau D, Pirson Y, Verellen-Dumoulin C, Macnicol A et al (1994) Intracranial aneurysms in autosomal dominant polycystic kidney disease. Kidney Int 45:1140-1146. https://doi.org/10.1038/ ki.1994.151

16. Chapman AB, Devuyst O, Eckardt K-U, Gansevoort RT et al (2015) Autosomal Dominant Polycystic Kidney Disease (ADPKD): executive summary from a Kidney Disease Improving Global Outcomes (KDIGO) Controversies Conference. Kidney Int 88:17-27. https://doi.org/10.1038/ki.2015.59

17. Dhar S, Tremmel M, Mocco J, Kim M et al (2008) Morphology Parameters for Intracranial Aneurysm Rupture Risk Assessment. Neurosurgery 63:185-197. https://doi.org/10.1227/01.neu.00003 16847.64140 .81

18. Wiebers DO, Whisnant JP, Huston J, Meissner I et al (2003) Unruptured intracranial aneurysms: natural history, clinical outcome, and risks of surgical and endovascular treatment. Lancet 362:103-110. https://doi.org/10.1016/s0140-6736(03)13860-3

19. Rozenfeld MN, Ansari SA, Shaibani A, Russell EJ et al (2014) Should Patients with Autosomal Dominant Polycystic Kidney Disease Be Screened for Cerebral Aneurysms? Am J Neuroradiol 35:3-9. https://doi.org/10.3174/ajnr.a3437

20. Fowler KJ (2019) An ADPKD Patient's View on Screening for Intracranial Aneurysms. Clin J Am Soc Nephrol 14:1117-1118. https://doi.org/10.2215/cjn.07460619

21. Molyneux A, Kerr R, Stratton I, Sandercock P et al (2002) International Subarachnoid Aneurysm Trial (ISAT) of neurosurgical clipping versus endovascular coiling in 2143 patients with ruptured intracranial aneurysms: a randomised trial. Lancet 360:12671274. https://doi.org/10.1016/s0140-6736(02)11314-6

22. Lee VW, Dexter MAJ, Mai J, Vladica P et al (2015) KHA-CARI Autosomal Dominant Polycystic Kidney Disease Guideline: Management of Intracranial Aneurysms. Semin Nephrol 35:612-617. e20. https://doi.org/10.1016/j.semnephrol.2015.10.014

23. Rozenfeld MN, Ansari SA, Mohan P, Shaibani A et al (2016) Autosomal Dominant Polycystic Kidney Disease and Intracranial Aneurysms: Is There an Increased Risk of Treatment? Am J Neuroradiol 37:290-293. https://doi.org/10.3174/ajnr.a4490

24. Levey AS, Pauker SG, Kassirer JP (1996) Occult Intracranial Aneurysms in Polycystic Kidney Disease - When is Cerebral Arteriography Indicated? N Engl J Med 308:986-994. https://doi. org/10.1056/nejm198304283081702

25. Flahault A, Trystram D, Fouchard M, Knebelmann B et al (2016) Screening for Unruptured Intracranial Aneurysms in Autosomal Dominant Polycystic Kidney Disease: A Survey of 420 
Nephrologists. PLoS ONE 11:e0153176. https://doi.org/10.1371/ journal.pone.0153176

26. Butler WE, Barker FG, Crowell RM (1996) Patients with Polycystic Kidney Disease Would Benefit from Routine Magnetic Resonance Angiographic Screening for Intracerebral Aneurysms: A Decision Analysis. Neurosurgery 38:506-516

27. Schrier RW, Belz MM, Johnson AM, Kaehny WD et al (2004) Repeat Imaging for Intracranial Aneurysms in Patients with Autosomal Dominant Polycystic Kidney Disease with Initially Negative Studies; A Prospective Ten-Year Follow-up. J Am Soc Nephrol 15:1023-1028. https://doi.org/10.1097/01.asn.00001 18527.74850 .66

28. Wilkinson DA, Heung M, Deol A, Chaudhary N et al (2018) Cerebral Aneurysms in Autosomal Dominant Polycystic Kidney Disease: A Comparison of Management Approaches. Neurosurgery 84:E352-E361. https://doi.org/10.1093/neuros/nyy336

29. Lasjaunias P, Wuppalapati S, Alvarez H, Rodesch G et al (2005) Intracranial aneurysms in children aged under 15 years: review of 59 consecutive children with 75 aneurysms. Childs Nerv Syst 21:437-450. https://doi.org/10.1007/s00381-004-1125-x

30. Buis DR, van Ouwerkerk WJR, Takahata H, Vandertop WP (2006) Intracranial aneurysms in children under 1 year of age: a systematic review of the literature. Childs Nerv Syst 22:1395-1409. https://doi.org/10.1007/s00381-006-0142-3

31. Allison JW, Davis PC, Sato Y, James CA et al (1998) Intracranial aneurysms in infants and children. Pediatr Radiol 28:223-229. https://doi.org/10.1007/s002470050336

32. Slator N, Talibi SS, Mundil N, Thomas A et al (2019) Paediatric intracranial aneurysms: a British institutional review. Childs Nerv Syst 35:1197-1205. https://doi.org/10.1007/s00381-019-04159-3

33. Thong KM, Ong ACM (2014) Sudden death due to subarachnoid haemorrhage in an infant with autosomal dominant polycystic kidney disease. Nephrol Dial Transplant 29:iv121-iv123. https:// doi.org/10.1093/ndt/gfu014

34. Kubo S, Nakajima M, Fukuda K, Nobayashi M et al (2004) A 4-year-old girl with autosomal dominant polycystic kidney disease complicated by a ruptured intracranial aneurysm. Eur J Pediatr 163:675-677. https://doi.org/10.1007/s00431-004-1528-4

35. Proesmans W, Damme BV, Casaer P, Marchal G (1982) Autosomal dominant polycystic kidney disease in the neonatal period: association with a cerebral arteriovenous. Pediatrics 70:971-975

36. Kääriäinen H, Koskimies O, Norio R (1988) Dominant and recessive polycystic kidney disease in children: evaluation of clinical features and laboratory data. Pediatr Nephrol 2:296-302. https:// doi.org/10.1007/bf00858681

37. Marlais M, Cuthell O, Langan D, Dudley J et al (2016) Hypertension in autosomal dominant polycystic kidney disease: a metaanalysis. Arch Dis Child 101:1142. https://doi.org/10.1136/archd ischild-2015-310221

38. Massella L, Mekahli D, Paripović D, Prikhodina L et al (2018) Prevalence of Hypertension in Children with Early-Stage ADPKD. Clin J Am Soc Nephrol 13:874-883. https://doi.org/10. 2215/cjn.11401017
39. Hiratsuka Y, Miki H, Kiriyama I, Kikuchi K et al (2008) Diagnosis of Unruptured Intracranial Aneurysms: 3T MR Angiography versus 64-channel Multi-detector Row CT Angiography. Magn Reson Med Sci 7:169-178. https://doi.org/10.2463/mrms.7.169

40. Arlachov Y, Ganatra RH (2012) Sedation/anaesthesia in paediatric radiology. Br J Radiol 85:e1018-e1031. https://doi.org/10.1259/ bjr/28871143

41. Malviya S, Voepel-Lewis T, Eldevik OP, Rockwell DT et al (2000) Sedation and general anaesthesia in children undergoing MRI and CT: adverse events and outcomes. Br J Anaesth 84:743-748. https://doi.org/10.1093/oxfordjournals.bja.a013586

42. Bhogal P, Pérez MA, Wendl C, Bäzner H et al (2017) Paediatric aneurysms - Review of endovascular treatment strategies. J Clin Neurosci 45:54-59. https://doi.org/10.1016/j.jocn.2017.08.009

43. Sorteberg A, Dahlberg D (2013) Intracranial Non-traumatic Aneurysms in Children and Adolescents. Curr Pediatr Rev 9:343-352. https://doi.org/10.2174/221155281120100005

44. Gimpel C, Bergmann C, Bockenhauer D, Breysem L et al (2019) International consensus statement on the diagnosis and management of autosomal dominant polycystic kidney disease in children and young people. Nat Rev Nephrol 15:713-726. https://doi.org/ 10.1038/s41581-019-0155-2

45. Bekelis K, Connolly ID, Do HM, Choudhri O (2016) Operative volume and outcomes of cerebrovascular neurosurgery in children. J Neurosurg Pediatrics 18:623-628. https://doi.org/10.3171/ 2016.5.peds 16137

46. Alawi A, Edgell RC, Elbabaa SK, Callison RC et al (2014) Treatment of cerebral aneurysms in children: analysis of the Kids' Inpatient Database: Clinical article. J Neurosurg Pediatrics 14:23-30. https://doi.org/10.3171/2014.4.peds13464

47. Dudley J, Winyard P, Marlais M, Cuthell O et al (2019) Clinical practice guideline monitoring children and young people with, or at risk of developing autosomal dominant polycystic kidney disease (ADPKD). BMC Nephrol 20:148. https://doi.org/10.1186/ s12882-019-1285-2

48. Trevethan R (2017) Sensitivity, Specificity, and Predictive Values: Foundations, Pliabilities, and Pitfalls in Research and Practice. Front Public Health 5:307. https://doi.org/10.3389/fpubh.2017. 00307

\section{Answers}

1. a; 2. d; 3. e; 4. c; 5. c and d

Publisher's note Springer Nature remains neutral with regard to jurisdictional claims in published maps and institutional affiliations. 